\title{
Application of Multi-Step Differential Transform Method on Flow of a Second-Grade Fluid over a Stretching or Shrinking Sheet
}

\author{
Mohammad Mehdi Rashidi, ${ }^{1,}$, Ali J. Chamkha ${ }^{3}$, Mohammad Keimanesh ${ }^{1}$ \\ ${ }^{1}$ Mechanical Engineering Department, Engineering Faculty, Bu-Ali Sina University, Hamedan, Iran \\ ${ }^{2}$ Department of Genius Mechanical, University of Sherbrooke, Sherbrooke, Canada \\ ${ }^{3}$ Manufacturing Engineering Department, The Public Authority for Applied Education and Training, Shuweikh, Kuwait \\ E-mail:mm_rashidi@yahoo.com \\ Received April 8, 2011; revised May 20, 2011; accepted June 1, 2011
}

\begin{abstract}
In this study, a reliable algorithm to develop approximate solutions for the problem of fluid flow over a stretching or shrinking sheet is proposed. It is depicted that the differential transform method (DTM) solutions are only valid for small values of the independent variable. The DTM solutions diverge for some differential equations that extremely have nonlinear behaviors or have boundary-conditions at infinity. For this reason the governing boundary-layer equations are solved by the Multi-step Differential Transform Method (MDTM). The main advantage of this method is that it can be applied directly to nonlinear differential equations without requiring linearization, discretization, or perturbation. It is a semi analytical-numerical technique that formulizes Taylor series in a very different manner. By applying the MDTM the interval of convergence for the series solution is increased. The MDTM is treated as an algorithm in a sequence of intervals for finding accurate approximate solutions for systems of differential equations. It is predicted that the MDTM can be applied to a wide range of engineering applications.
\end{abstract}

Keywords: Non-Newtonian Fluid, Stretching Surface, Shrinking Sheet, Multi-Step Differential Transform Method (MDTM)

\section{Introduction}

A number of industrially important fluids such as molten plastics, polymer solutions, pulps, foods and slurries, fossil fuels, special soap solutions, blood, paints, certain oils and greases display a rheologically-complex non-Newtonian fluid behavior. Non-Newtonian fluids exhibit a non-linear relationship between shear stress and shear rate. The Navier-Stokes equations governing the flow of these fluids are complicated due to their highly non-linear nature. The non-linearity nature of the equations comes from the constitutive equations which represents the material properties of rheological fluids. It is, therefore, not easy to find their exact solutions because the superposition principle for non-linear partial differential equations does not hold. Numerous models have been developed to simulate a wide variety of rheological fluids, including viscoelastic differential models [1], couple stress fluid models [2] and micropolar fluid models [3].
Magnetohydrodynamic flows also arise in many applications including materials processing [4] and MagnetoHydro-Dynamic (MHD) energy generators [5].

Boundary-layer flows of non-Newtonian fluids have been of great interest to researchers during the past three decades. These investigations were for non-Newtonian fluids of the differential type [6]. In the case of fluids of differential type, the equations of motion are of order higher than that of the Navier-Stokes equations, and thus, the adherence boundary condition is insufficient to determine the solution completely [7-9]. The same is also true for the boundary-layer approximations of the equations of motion.

In this paper, a reliable algorithm of the DTM, namely MDTM [10] is used to explain the behavior of the fluid such as stream function profile, velocity profile and variations of the velocity profile.

The concept of the DTM was first introduced by Zhou [11] in 1986 and it was used to solve both linear and 
non-linear initial-value problems in electric circuit analysis. This method constructs, for differential equations, an analytical solution in the form of a polynomial. Not like the traditional high-order Taylor series method that requires symbolic computation, the DTM is an iterative procedure for obtaining Taylor series solutions [12,13]. Against these advantages, the DTM solutions diverge for some highly non-linear differential equations that have boundary conditions at infinity [14].

The paper has been organized as follows: In Section 2, the basic concepts of the differential transform method are presented. The basic concepts of the multi-step differential transform method are presented in Section 3. In Section 4, the mathematical formulation is introduced. The analytical solution by the MDTM is presented in Section 5. Section 6 contains the results and their discussion. Finally, the conclusions are summarized in Section 7.

\section{Basic Concepts of the Differential Transform Method}

Transformation of the $k$ th derivative of a function in one variable is as follows [15]

$$
F(k)=\frac{1}{k !}\left[\frac{\mathrm{d}^{k} f(t)}{\mathrm{d} t^{k}}\right]_{t=t_{0}}
$$

and the inverse transformation is defined by

$$
f(t)=\sum_{k=0}^{\infty} F(k)\left(t-t_{0}\right)^{k}
$$

From Equations (1) and (2), we get:

$$
f(t)=\left.\sum_{k=0}^{\infty} \frac{\left(t-t_{0}\right)^{k}}{k !} \frac{\mathrm{d}^{k} f(t)}{\mathrm{d} t^{k}}\right|_{t=t_{0}}
$$

which implies that the concept of the differential transform method is resulting from Taylor series expansion, but the method does not calculate the derivatives representatively. However, the relative derivatives are calculated by an iterative way which is described by the transformed equations of the original function. For implementation purposes, the function $f(t)$ is expressed by a finite series and Equation (2) can be written as

$$
f(t) \cong \sum_{k=0}^{i} F(k)\left(t-t_{0}\right)^{k},
$$

where $F(k)$ is the differential transform of $f(t)$.

\section{Basic Concepts of the Multi-Step Differential Transform Method}

When the DTM is used for solving differential equations with the boundary condition ns at infinity or problems that have highly non-linear behavior, the obtained results were found to be incorrect (when the boundary-layer variable go to infinity, the obtained series solutions are divergent). Besides that, power series are not useful for large values of the independent variable.

To overcome this shortcoming, the multi-step DTM that has been developed for the analytical solution of the differential equations is presented in this section. For this purpose, the following non-linear initial-value problem is considered,

$$
u\left(t, f, f^{\prime}, \cdots, f^{(p)}\right)=0,
$$

subject to the initial conditions $f^{(k)}(0)=c_{k}$, for $k=0$, $1, \cdots, p-1$.

Let $[0, T]$ be the interval over which we want to find the solution of the initial-value problem (5). In actual applications of the DTM, the approximate solution of the initial value problem (5) can be expressed by the following finite series:

$$
f(t)=\sum_{n=0}^{N} a_{n} t^{n} \quad t \in[0, T] .
$$

The multi-step approach introduces a new idea for constructing the approximate solution. Assume that the interval $[0, T]$ is divided into $M$ subintervals $\left[t_{m-1}\right.$, $\left.t_{m}\right], \quad m=1,2, \cdots, M$ of equal step size $h=T / M$ by using the nodes $t=m h$. The main ideas of the multi-step DTM are as follows. First, we apply the DTM to Equation (5) over the interval $\left[0, t_{1}\right]$, we will obtain the following approximate solution,

$$
f_{1}(t)=\sum_{n=0}^{K} a_{1 n} t^{n} \quad t \in\left[0, t_{1}\right],
$$

using the initial conditions $f_{1}^{(k)}(0)=c_{k}$. For $m \geq 2$ and at each subinterval $\left[t_{m-1}, t_{m}\right]$ we will use the initial conditions $f_{m}^{(k)}\left(t_{m-1}\right)=f_{m-1}^{(k)}\left(t_{m-1}\right)$ and apply the DTM to Equation (5) over the interval $\left[t_{m-1}, t_{m}\right]$, where $t_{0}$ in Equation (1) is replaced by $t_{m-1}$. The process is repeated and generates a sequence of approximate solutions $f_{m}(t), m=1,2, \cdots, M$, for the solution $f(t)$,

$$
f_{m}(t)=\sum_{n=0}^{K} a_{m n}\left(t-t_{m-1}\right)^{n}, t \in\left[t_{m}, t_{m+1}\right],
$$

where $N=K \cdot M$. In fact, the multi-step DTM assumes the following solution:

$$
f(t)=\left\{\begin{array}{l}
f_{1}(t), t \in\left[0, t_{1}\right] \\
f_{2}(t), t \in\left[t_{1}, t_{2}\right] \\
n \\
f_{M}(t), t \in\left[t_{M-1}, t_{M}\right]
\end{array} .\right.
$$

The new algorithm, multi-step DTM, is simple for computational performance for all values of $h$. It is 


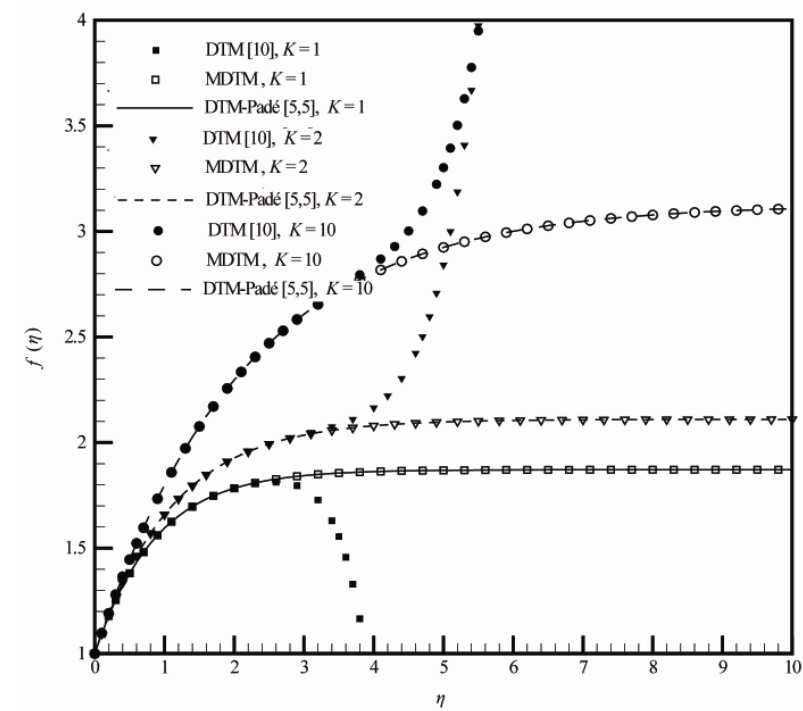

Figure 1. Variation $f(\eta)$ with respect to $K$, when $M=2$, $s=1, a=1$, for the stretching sheet.

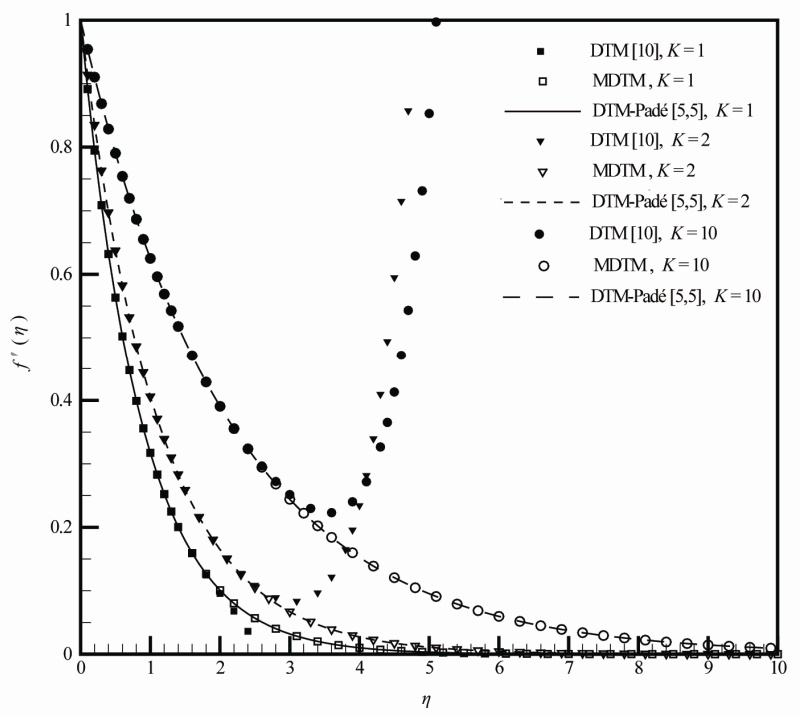

Figure 2. Variation $f^{\prime}(\eta)$ with respect to $K$, when $M=2$, $s=1, a=1$, for the stretching sheet.

easily observed that if the step size $h=T$, then the multi-step DTM reduces to the classical DTM. As we will see in the next section, the main advantage of the new algo-rithm is that the obtained series solution converges for wide time regions and can approximate non-chaotic or chaotic solutions.

\section{Mathematical Formulation}

Consider the flow of a second-order fluid following Equations (10-12) as

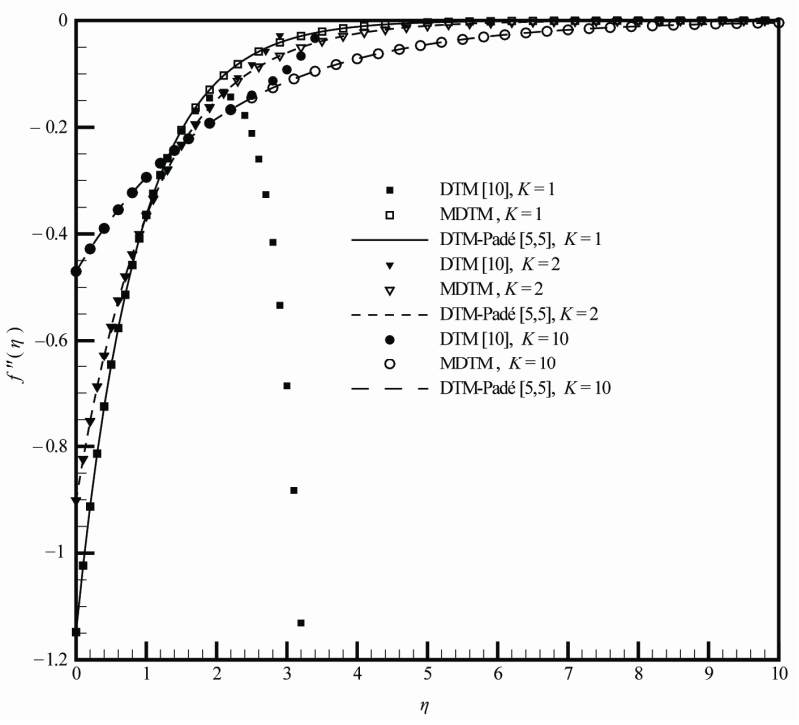

Figure 3. Variation $f^{\prime \prime}(\eta)$ with respect to $K$, when $M=2, s=1, a=1$, for the stretching sheet.

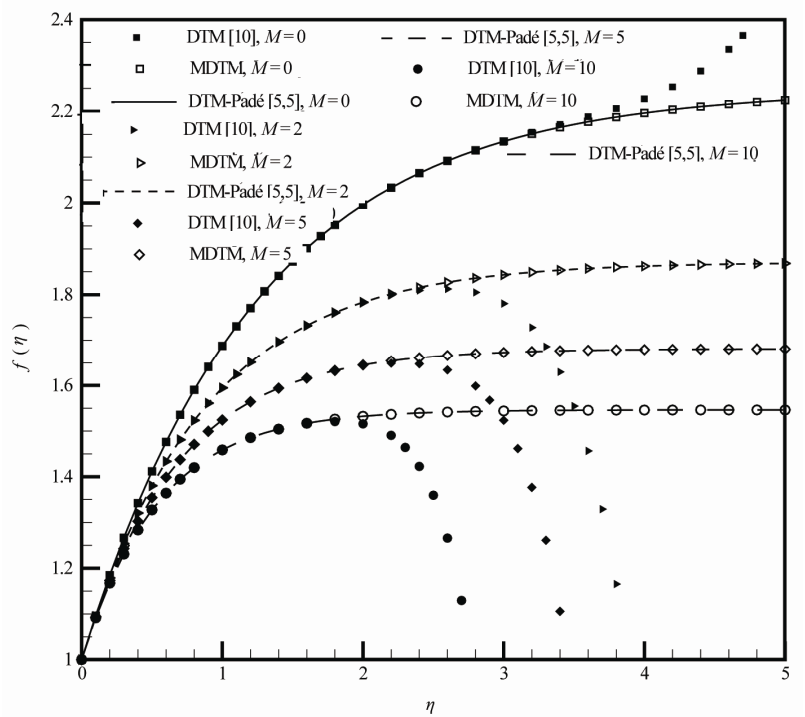

Figure 4. Variation $f(\eta)$ with respect to $M$, when $K=1, s=1, a=1$, for the stretching sheet.

$$
T=-p I+\mu \mathrm{A}_{1}+\alpha_{1} \mathrm{~A}_{2}+\alpha_{2} \mathrm{~A}_{1}^{2},
$$

where $\mathrm{A}_{1}=($ gradv $)+(\text { gradv })^{T}$, and

$$
\begin{gathered}
\mathrm{A}_{2}=\mathrm{dA}_{1} / \mathrm{d} t+\mathrm{A}_{1}(\text { gradv) })^{T} \mathrm{~A}_{1}, \\
\mu \geq 0, \quad \alpha_{1} \geq 0, \quad \alpha_{1}+\alpha_{2}=0 .
\end{gathered}
$$

The flow past a flat sheet coinciding with the plane $y=0$, the flow being confined to $y>0$. Two equal and opposite forces are applied along the $x$-axis so that the wall is stretched, keeping the origin fixed, and a uniform magnetic field $B_{0}$ is imposed along the $y$-axis. The steady two-dimensional boundary-layer equations for 


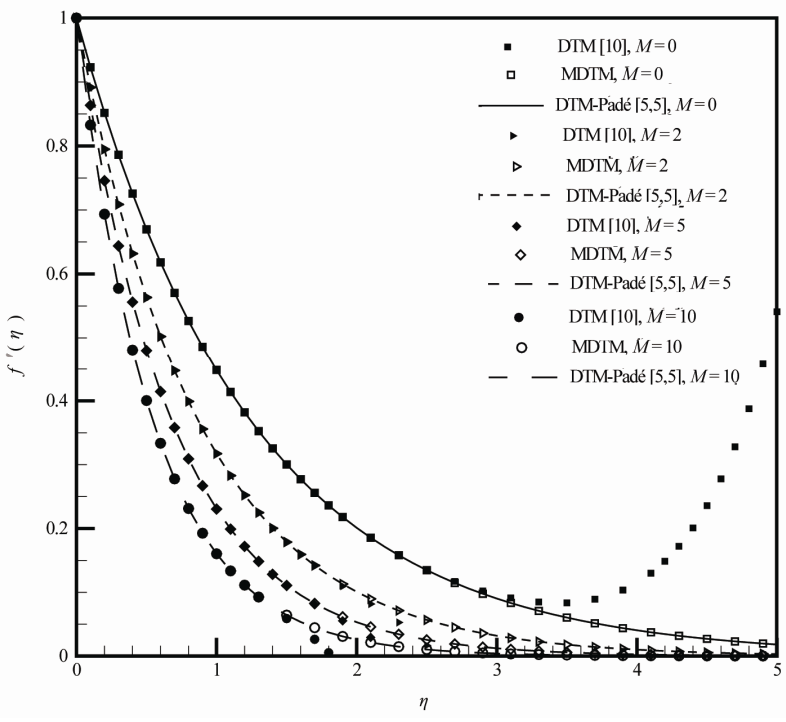

Figure 5. Variation $f^{\prime}(\eta)$ with respect to $M$, when $K=1, s=1, a=1$, for the stretching sheet.

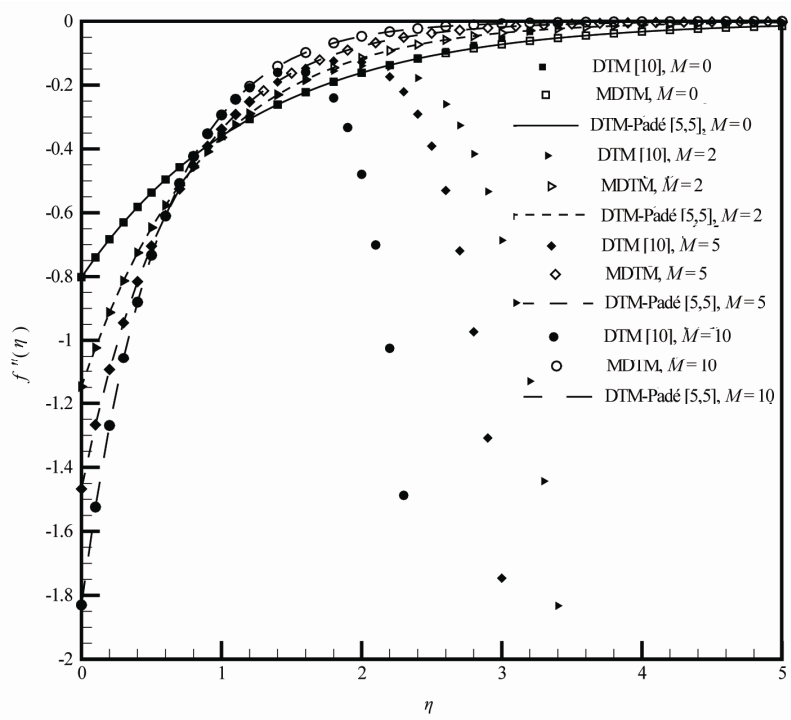

Figure 6. Variation $f^{\prime \prime}(\eta)$ with respect to $M$, when $K=1, s=1, a=1$, for the stretching sheet.

this fluid in the primitive usual notation are

$$
\begin{gathered}
\frac{\partial u}{\partial x}+\frac{\partial v}{\partial y}=0 \\
u \frac{\partial u}{\partial x}+v \frac{\partial v}{\partial y}=v \frac{\partial^{2} u}{\partial y^{2}}-\frac{\sigma_{0} B_{0}^{2}}{\rho} u \\
+\lambda\left[\frac{\partial}{\partial x}\left(u \frac{\partial^{2} u}{\partial y^{2}}\right)+\frac{\partial u}{\partial y} \frac{\partial^{2} u}{\partial x \partial y}+v \frac{\partial^{3} u}{\partial y^{3}}\right]
\end{gathered}
$$

The precise mathematical problem considered is [16]

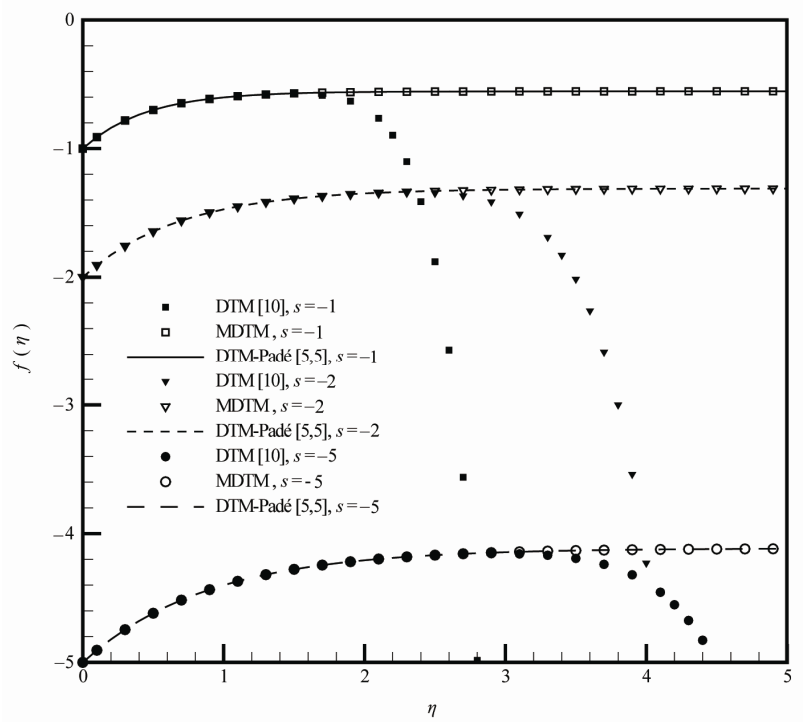

Figure 7. Variation $f(\eta)$ with respect to $s$, when $K=1$, $M=0, a=1$, for the stretching sheet.

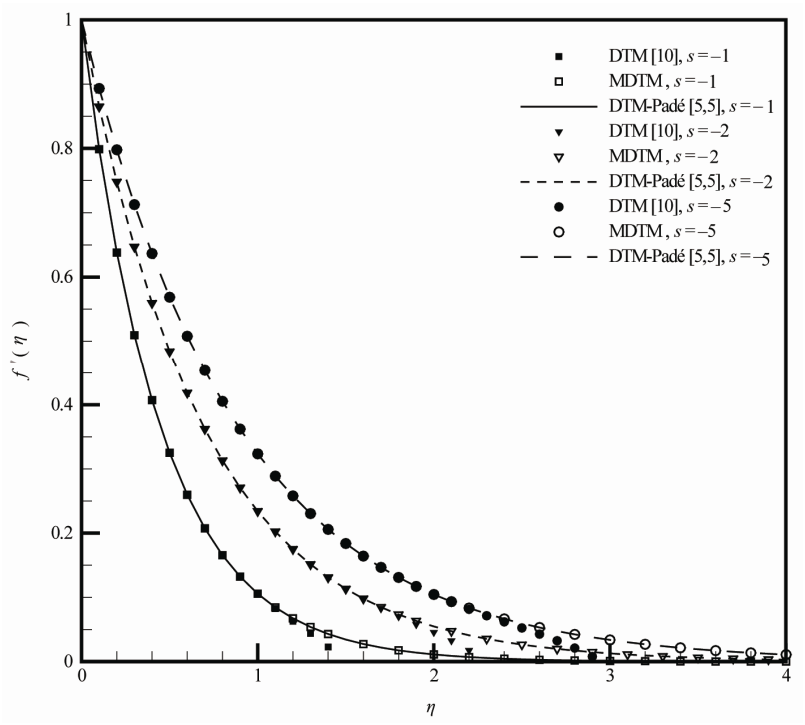

Figure 8. Variation $f^{\prime}(\eta)$ with respect to $s$, when $K=1$, $M=0, a=1$, for the stretching sheet.

$$
\left(f^{\prime}\right)^{2}-f f^{\prime \prime}+M f^{\prime}=f^{\prime \prime \prime}+K\left[2 f^{\prime} f^{\prime \prime \prime}-\left(f^{\prime \prime}\right)^{2}-f f^{i v}\right]
$$

The appropriate boundary conditions for the problem are

$$
\begin{gathered}
f(0)=s, \quad f^{\prime}(0)=a, \\
f^{\prime}(\infty) \rightarrow 0, \quad f^{\prime \prime}(\infty) \rightarrow 0 .
\end{gathered}
$$

\section{Analytical Solution be the MDTM}

Applying the MDTM to Equation (15) gives the following recursive relation in each sub-domain $\left(t_{i}, t_{i+1}\right), i=0$, $1, n, N-1$. 


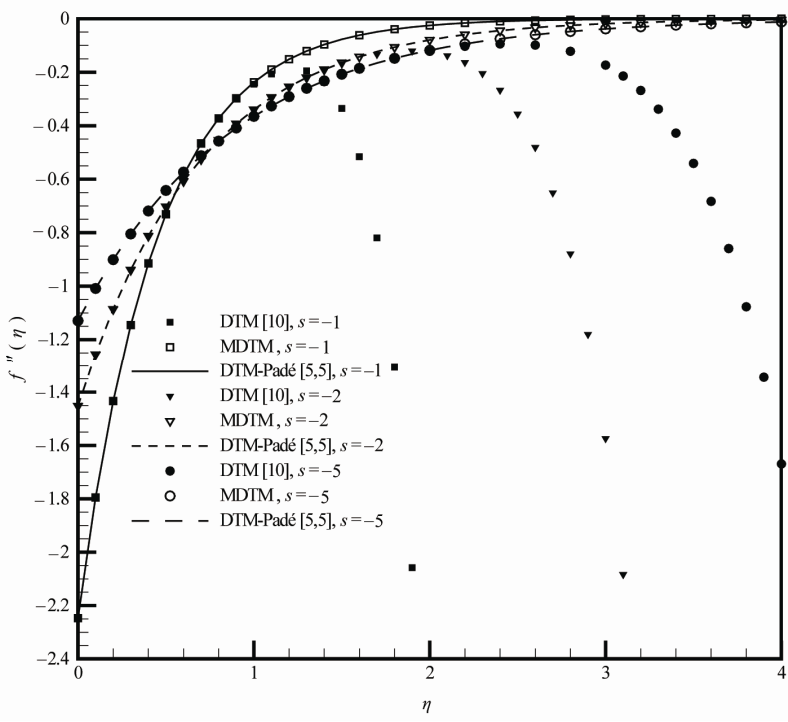

Figure 9. Variation $f^{\prime \prime}(\eta)$ with respect to $s$, when $K=1$, $M=0, a=1$, for the stretching sheet.

Table 1. The operations for the one-dimensional DTM.

\begin{tabular}{cc}
\hline Original function & Transformed function \\
\hline$f(t)=u(t) \pm v(t)$ & $F(k)=U(k) \pm V(k)$ \\
$f(t)=\lambda u(t)$ & $F(k)=\lambda U(k)$ \\
$f(t)=\frac{\mathrm{d}^{n} u(t)}{\mathrm{d} t^{n}}$ & $F(k)=\frac{(k+n) !}{k !} U(k+n)$ \\
$f(t)=\frac{\mathrm{d} u(t)}{\mathrm{d} t} \frac{\mathrm{d} u(t)}{\mathrm{d} t}$ & $F(k)=\sum_{r=0}^{k}(r+1)(k-r+1) U(r+1) U(k-r+1)$ \\
$f(t)=\frac{\mathrm{d}^{2} u(t)}{\mathrm{d} t^{2}} \frac{\mathrm{d}^{2} u(t)}{\mathrm{d} t^{2}}$ & $F(k)=\sum_{r=0}^{k}(k+r+1)(r+2)(k-r+2)$ \\
$f(t)=u(t) \frac{\mathrm{d}^{2} u(t)}{\mathrm{d} t^{2}}$ & $F(k)=\sum_{r=0}^{k}(k-r+2)(k-r+1) U(r) U(k-r+2)$ \\
$f(t)=u(t) \frac{\mathrm{d} v(t)}{\mathrm{d} t}$ & $F(k)=\sum_{r=0}^{k}(k-r+1) U(r) V(k-r+1)$. \\
\hline
\end{tabular}

$$
\begin{gathered}
(k+1)(k+2)(k+3) F(k+3) \\
+\sum_{r=0}^{k} F(r) F(k-r+2)(k-r+1)(k-r+2) \\
-\sum_{r=0}^{k} F(r+1) F(k-r+1)(r+1)(k-r+1) \\
+k\left(\begin{array}{c}
2 \sum_{r=0}^{k} F(k+1) F(k-r+3)(r+1) \\
-\sum_{r=0}^{k} F(r+2) F(k-r+2)(k-r+1)(k-r+2) \\
-\sum_{r=0}^{k} F(r) F(k-r+4)(k-r+2)(k-r+3)(k-r+4)
\end{array}\right),
\end{gathered}
$$

where $F(k)$ is the differential transforms of $f(\eta)$.

We can consider the boundary conditions [Equations. (15) and (16)] as follows:

$$
\begin{aligned}
f(0) & =s, \quad f^{\prime}(0)=a, \\
f^{\prime \prime}(0) & =\alpha, \quad f^{\prime \prime \prime}(0)=\beta .
\end{aligned}
$$

The differential transform of the above initial conditions are as follows

$$
\begin{gathered}
F(0)=s, \quad F(1)=a, \\
F(2)=\alpha / 2, \quad F(3)=\beta / 6 .
\end{gathered}
$$

Moreover, substituting Equations (20) and (21) into Equation (17) and by using the recursive method, we can calculate other values of $F(k)$. Hence, substituting all $F(k)$, into Equation (4), we obtain series solutions. By

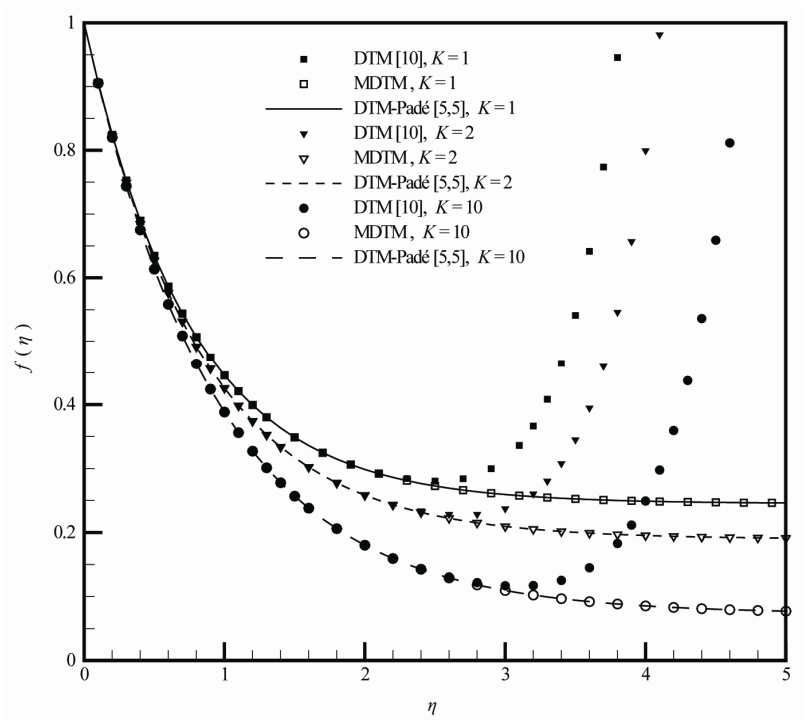

Figure 10. Variation $f(\eta)$ with respect to $K$, when $M=2, s=1, a=-1$, for the shrinking sheet.

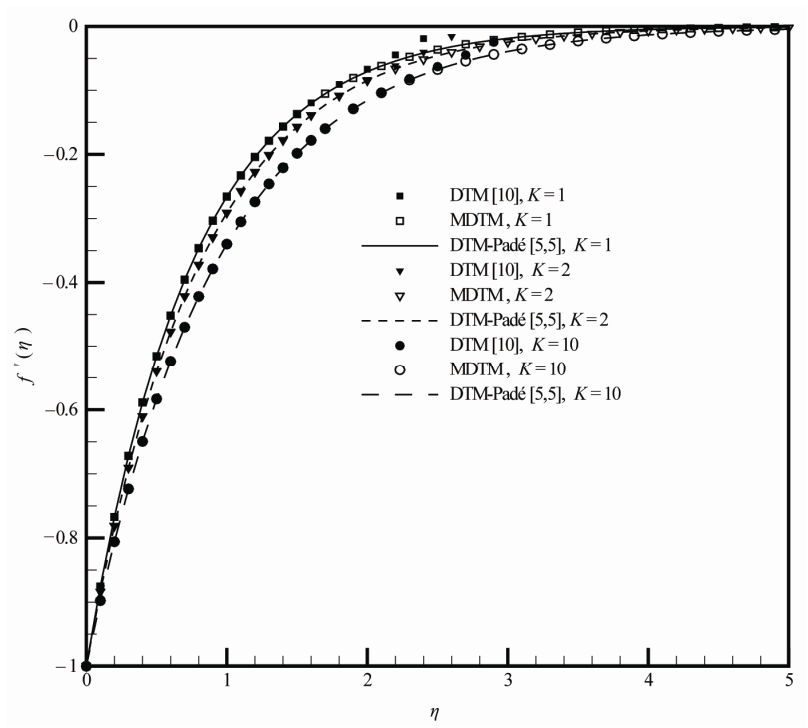

Figure 11. Variation $f^{\prime}(\eta)$ with respect to $K$, when $M=2, s=1, a=-1$, for the shrinking sheet. 
Table 2. Comparison of obtained results for $f^{\prime}(\eta)$, when $M=2, s=1$ and $a=1$ and various values of parameter $K$.

\begin{tabular}{|c|c|c|c|c|c|c|}
\hline \multirow{3}{*}{$\eta$} & \multicolumn{6}{|c|}{$f^{\prime}(\eta)$} \\
\hline & \multicolumn{3}{|c|}{$K=1}$. & \multicolumn{3}{|c|}{$K=2$} \\
\hline & DTM & Padé $[5,5]$ & MDTM & DTM & Padé $[5,5]$ & MDTM \\
\hline 0.0 & 1.00000 & 1.00000 & 1.00000 & 1.00000 & 1.00000 & 1.00000 \\
\hline 0.5 & 0.716312 & 0.706233 & 0.706233 & 0.761281 & 0.747084 & 0.747084 \\
\hline 1.0 & 0.558208 & 0.498765 & 0.498765 & 0.643042 & 0.558134 & 0.558134 \\
\hline 1.5 & 0.507194 & 0.352244 & 0.352244 & 0.641502 & 0.416973 & 0.416973 \\
\hline 2.0 & 0.549619 & 0.248766 & 0.248766 & 0.754462 & 0.311513 & 0.311513 \\
\hline 2.5 & 0.671554 & 0.175687 & 0.175687 & 0.975226 & 0.232727 & 0.232727 \\
\hline 3.0 & 0.779422 & 0.124076 & 0.124076 & 1.16384 & 0.173866 & 0.173866 \\
\hline 3.5 & 0.467092 & 0.0876271 & 0.0876264 & 0.645226 & 0.129893 & 0.129893 \\
\hline 4.0 & -1.51143 & 0.0618864 & 0.0618847 & -2.73495 & 0.0970411 & 0.0970406 \\
\hline 4.5 & -8.28107 & 0.0437091 & 0.043705 & -14.5163 & 0.0724986 & 0.0724975 \\
\hline 5.0 & -26.7055 & 0.0308744 & 0.0308659 & -47.0937 & 0.0541641 & 0.0541617 \\
\hline 5.5 & -70.4832 & 0.0218145 & 0.0217985 & -125.568 & 0.0404682 & 0.0404633 \\
\hline 6.0 & -165.017 & 0.0154227 & 0.0153948 & -297.038 & 0.0302383 & 0.0302295 \\
\hline 6.5 & -354.731 & 0.0109179 & 0.0108723 & -644.682 & 0.022599 & 0.0225839 \\
\hline 7.0 & -713.66 & 0.00774892 & 0.00767839 & -1308.26 & 0.0168963 & 0.0168721 \\
\hline 7.5 & -1360.3 & 0.00552703 & 0.00542273 & -2513.06 & 0.0126421 & 0.0126049 \\
\hline 8.0 & -2477.92 & 0.00397796 & 0.00382971 & -4609.63 & 0.00947179 & 0.00941688 \\
\hline 8.5 & -4341.63 & 0.0029083 & 0.00270467 & -8127.06 & 0.00711334 & 0.0070352 \\
\hline 9.0 & -7353.9 & 0.00218163 & 0.00191012 & -13843.1 & 0.00536369 & 0.00525588 \\
\hline 9.5 & -12090.3 & 0.00170165 & 0.00134899 & -22874.4 & 0.00407131 & 0.00392658 \\
\hline 10 & -19357.2 & 0.00140031 & 0.000952703 & -36792.3 & 0.00312311 & 0.00293349 \\
\hline
\end{tabular}

Table 3. Comparison of obtained results for $-f^{\prime \prime}(\eta)$, when $M=2, s=1$ and $a=1$ and various values of parameter $K$.

\begin{tabular}{|c|c|c|c|c|c|c|}
\hline \multirow{3}{*}{$\eta$} & \multicolumn{6}{|c|}{$-f^{\prime \prime}(\eta)$} \\
\hline & \multicolumn{3}{|c|}{$K=1$} & \multicolumn{3}{|c|}{$K=2$} \\
\hline & DTM & Padé $[5,5]$ & MDTM & DTM & Padé $[5,5]$ & MDTM \\
\hline 0.0 & 0.695621 & 0.695621 & 0.695621 & 0.583156 & 0.583156 & 0.583156 \\
\hline 0.5 & 0.437262 & 0.49127 & 0.49127 & 0.359195 & 0.435667 & 0.435667 \\
\hline 1.0 & 0.202525 & 0.346951 & 0.346951 & 0.116804 & 0.325479 & 0.325479 \\
\hline 1.5 & 0.00605472 & 0.245028 & 0.245028 & -0.109835 & 0.24316 & 0.24316 \\
\hline 2.0 & -0.173392 & 0.173047 & 0.173047 & -0.343373 & 0.181661 & 0.181661 \\
\hline 2.5 & -0.290258 & 0.122211 & 0.122211 & -0.505173 & 0.135716 & 0.135716 \\
\hline 3.0 & -0.0223928 & 0.0863097 & 0.0863097 & -0.0537612 & 0.101391 & 0.101391 \\
\hline 3.5 & 1.65387 & 0.0609548 & 0.0609548 & 2.78564 & 0.0757477 & 0.0757477 \\
\hline 4.0 & 7.24184 & 0.0430483 & 0.0430482 & 12.4698 & 0.0565899 & 0.0565899 \\
\hline 4.5 & 22.024 & 0.0304023 & 0.0304021 & 38.5972 & 0.0422774 & 0.0422774 \\
\hline 5.0 & 56.0808 & 0.0214713 & 0.0214709 & 99.7758 & 0.0315848 & 0.0315847 \\
\hline 5.5 & 127.258 & 0.0151642 & 0.0151635 & 229.359 & 0.0235966 & 0.0235964 \\
\hline 6.0 & 265.338 & 0.0107103 & 0.010709 & 483.575 & 0.0176288 & 0.0176285 \\
\hline 6.5 & 517.708 & 0.00756533 & 0.00756301 & 952.633 & 0.0131705 & 0.01317 \\
\hline 7.0 & 956.839 & 0.00534496 & 0.00534125 & 1775.47 & 0.00984004 & 0.00983907 \\
\hline 7.5 & 1689.93 & 0.00377781 & 0.00377216 & 3158.83 & 0.00735216 & 0.0073506 \\
\hline 8.0 & 2871.07 & 0.00267225 & 0.00266403 & 5401.49 & 0.00549389 & 0.00549152 \\
\hline 8.5 & 4716.41 & 0.00189295 & 0.00188142 & 8924.45 & 0.0041061 & 0.00410262 \\
\hline 9.0 & 7522.61 & 0.00134434 & 0.00132872 & 14308. & 0.00306991 & 0.003065 \\
\hline 9.5 & 11689.3 & 0.000958921 & 0.000938387 & 22336.5 & 0.00229654 & 0.00228981 \\
\hline 10 & 17745.6 & 0.000689019 & 0.00066272 & 34052.4 & 0.00171964 & 0.00171068 \\
\hline
\end{tabular}

using the asymptotic boundary condition $F(2)=\alpha / 2$ and $f^{\prime \prime}(\infty) \rightarrow 0$, we can obtain $\alpha, \beta$. For analytical solution of the considered problem, the convergence analysis was performed and in Equation (4), the $i$ value is selected equal to 10 and the interval was set equal to 0.01 .

\section{Results and Discussion}

Equation (15) with transformed boundary conditions was solved analytically using the DTM and the MDTM. In order to give a comprehensive approach of the problem, a comparison between DTM, MDTM and DTM-Padé solutions for various parameters is presented.

In Figures 1-9, solutions for the stretching sheet are illustrated. Figures 1-3 show the variation of $f(\eta)$, $f^{\prime}(\eta), f^{\prime \prime}(\eta)$ for various values of $K$. It is observed that increasing the parameter $K$. causes increases in $f(\eta), f^{\prime}(\eta)$, profiles, respectively. The influence of the magnetic parameter $M$, on $f(\eta), f^{\prime}(\eta), f^{\prime \prime}(\eta)$ 


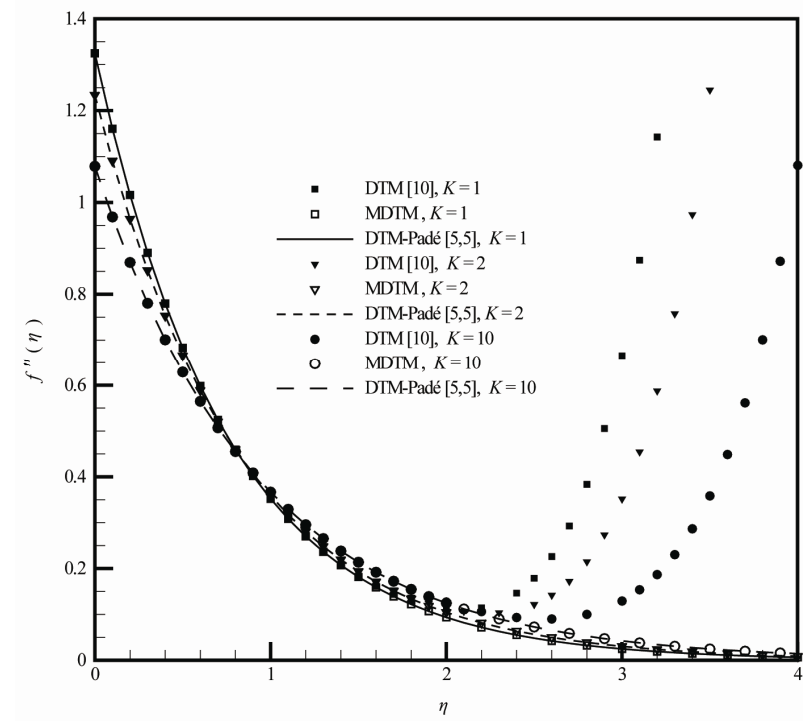

Figure 12. Variation $f^{\prime \prime}(\eta)$ with respect to $K$, when $M=2, s=1, a=-1$, for the shrinking sheet.

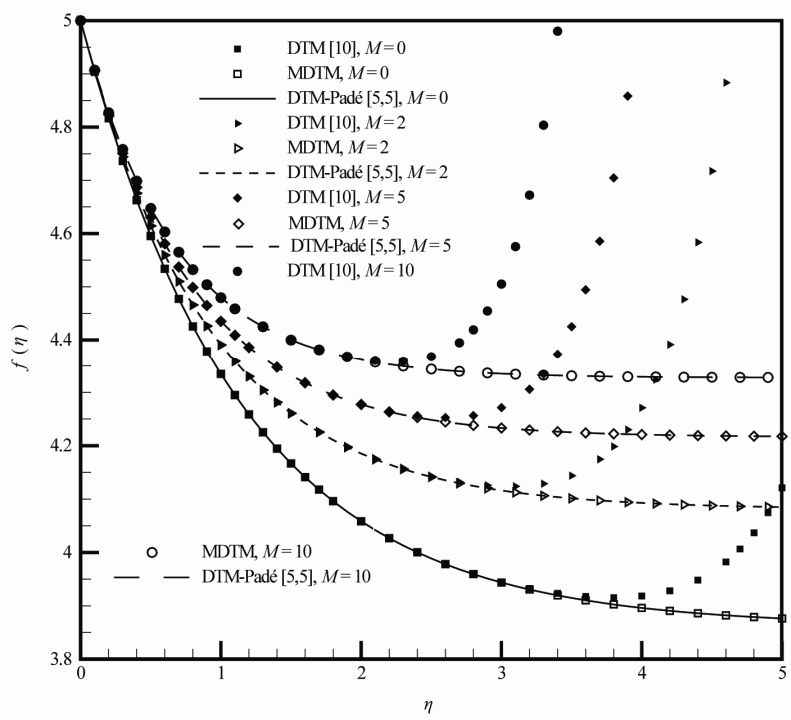

Figure 13. Variation $f(\eta)$ with respect to $M$, when $K=1, s=5, a=-1$, for the shrinking sheet.

is presented in Figures 4-6. It can be concluded that, an increase in the magnetic parameter decreases $f(\eta)$, $f^{\prime}(\eta)$, profiles. The effects of the parameter $s$ are shown in Figures 7-9. It is clear that an increase in this parameter causes a remarkable rise in $f(\eta)$ profile. Conversely, an increase in the value of $S$, causes a corresponding decrease in $f^{\prime}(\eta)$ profile.

In Figures 10-18, solutions for the shrinking sheet are shown. Figures 10-12 show the variations of $f(\eta)$, $f^{\prime}(\eta), f^{\prime \prime}(\eta)$ for various values of $K$. It is observed that increasing the parameter $K$. causes decreases in $f(\eta), f^{\prime}(\eta)$, profiles, respectively. The influence of

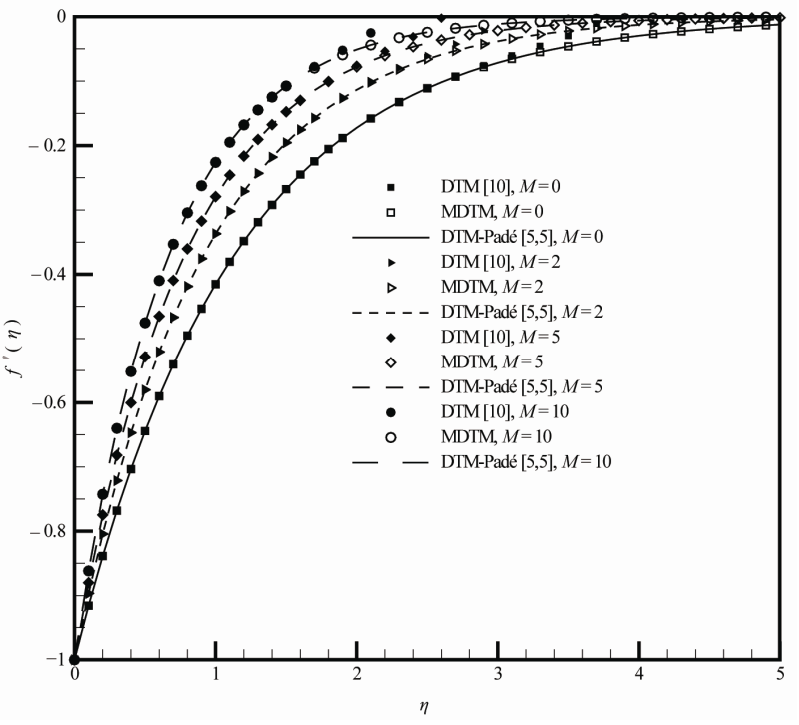

Figure 14. Variation $f^{\prime}(\eta)$ with respect to $M$, when $K=1, s=5, a=-1$, for the shrinking sheet.

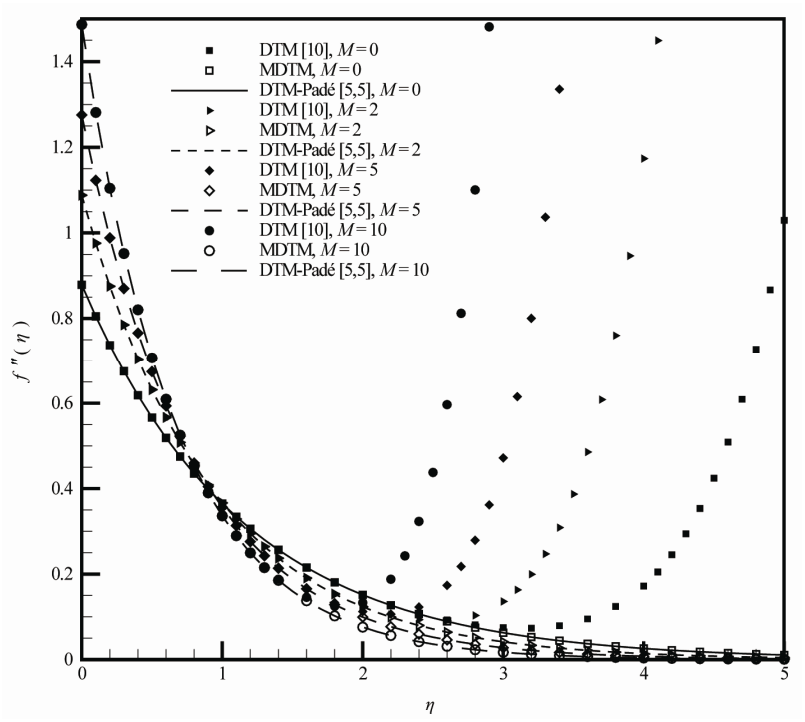

Figure 15. Variation $f^{\prime \prime}(\eta)$ with respect to $M$, when $K=1, s=5, a=-1$, for the shrinking sheet.

the magnetic parameter $M$, on $f(\eta), f^{\prime}(\eta), f^{\prime \prime}(\eta)$ is presented in Figures 13-15. It can be concluded that, an increase in the magnetic parameter increases $f(\eta)$,

In Figures 10-18, solutions for the shrinking sheet are shown. Figures 10-12 show the variations of $f(\eta)$, $f^{\prime}(\eta), \quad f^{\prime \prime}(\eta)$ for various values of $K$. It is observed that increasing the parameter $K$. causes decreases in $f(\eta), f^{\prime}(\eta)$, profiles, respectively. The influence of the magnetic parameter $M$, on $f(\eta), f^{\prime}(\eta), f^{\prime \prime}(\eta)$ is presented in Figures 13-15. It can be concluded that, an increase in the magnetic parameter increases $f(\eta)$, $f^{\prime}(\eta), f^{\prime \prime}(\eta)$ profiles. The effects of the parameter 


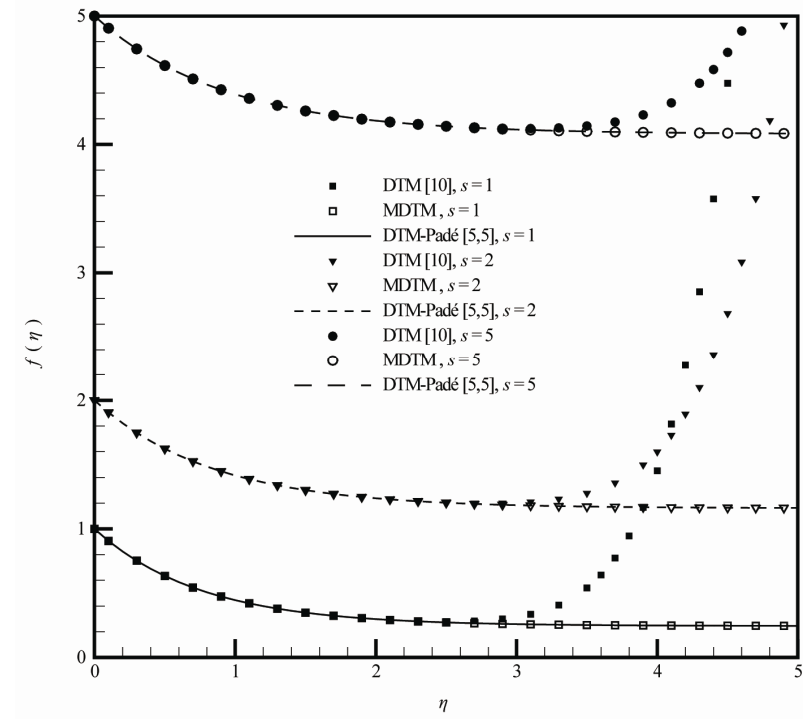

Figure 16. Variation $f(\eta)$ with respect to $s$, when $K=1$, $M=2, a=-1$, for the shrinking sheet.

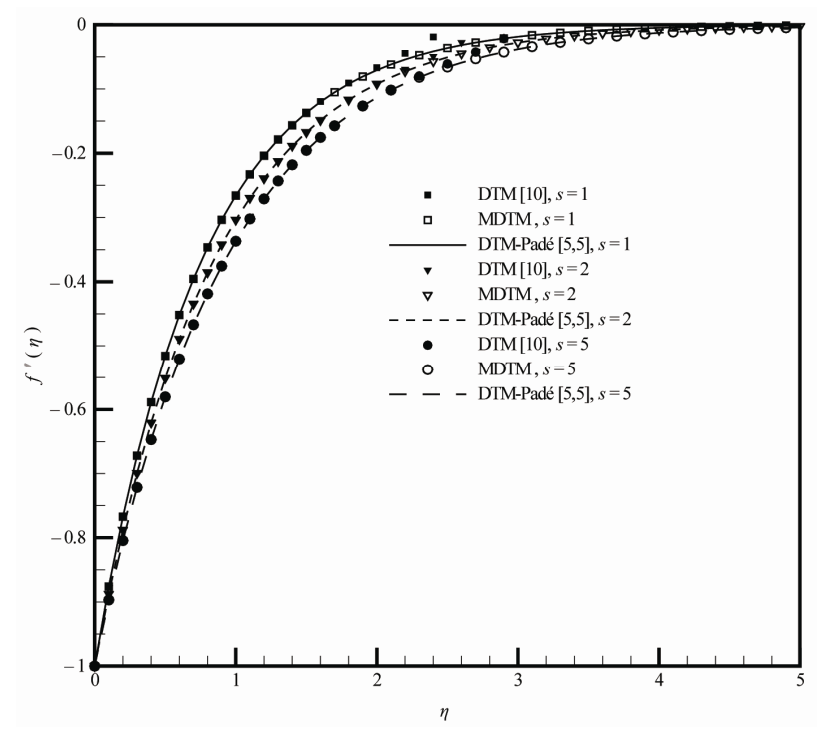

Figure 17. Variation $f^{\prime}(\eta)$ with respect to $s$, when $K=1, M=2, a=-1$, for the shrinking sheet.

$S$, are shown in Figures 16-18. It is clear that increasing this parameter causes a remarkable rise in $f(\eta)$ profile. Conversely, increasing the value of $s$, causes a decrease in $f^{\prime}(\eta)$ profile. The Residual errors for Equation 15 are plotted in Figures 19-20 using the DTM and MDTM respectively. In order to verify the efficiency of the proposed method in comparison with the DTM and DTM-Padé solutions, we report the obtained results in Tables 2 and $\mathbf{3}$ for $f(\eta)$ and $f^{\prime}(\eta)$ when $M=2, s=1$ and $x=1$ and various values of the parameter $K$. for $f^{\prime}(\eta)$ and $-f^{\prime \prime}(\eta)$, respectively. It is obvious from Tables $\mathbf{2}$ and $\mathbf{3}$ that the MDTM is a

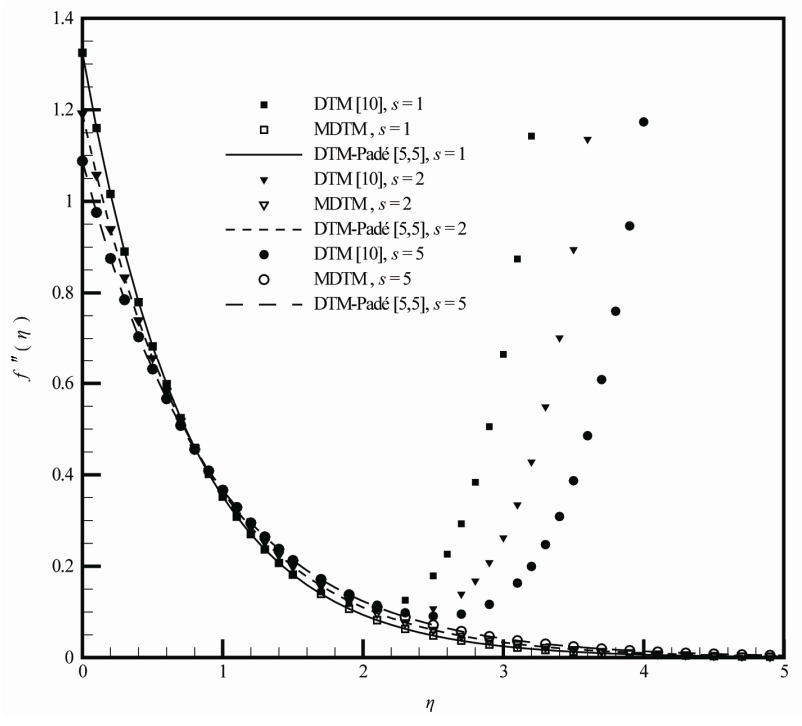

Figure 18. Variation $f^{\prime \prime}(\eta)$ with respect to $s$, when $K=1, M=2, a=-1$, for the shrinking sheet.

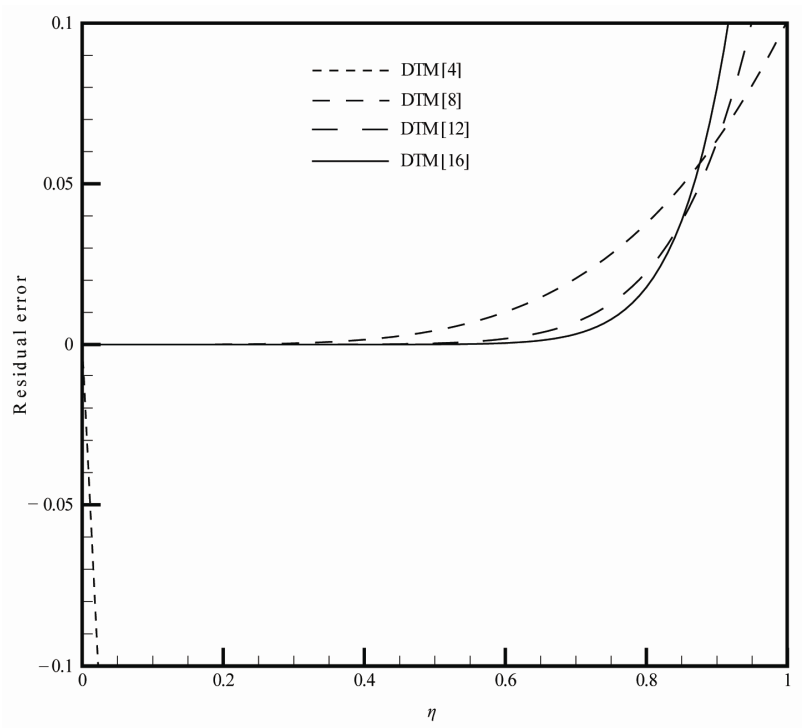

Figure 19. Residual error for Equation (15) using the DTM approximations when $K=1, M=2, s=5$ and $a=-1$.

reliable algorithm method.

\section{Conclusions}

In this paper, the Multi-step differential transform method was applied successfully to find the analytical solution of resulting ordinary differential equation for the problem of flow of a second-grade fluid over a stretching or shrinking sheet. The present method reduces the computational difficulties of the other methods (same as the HAM, VIM, ADM and HPM) [17-19], on the other hand, this method has some limitations (respect to HAM, 


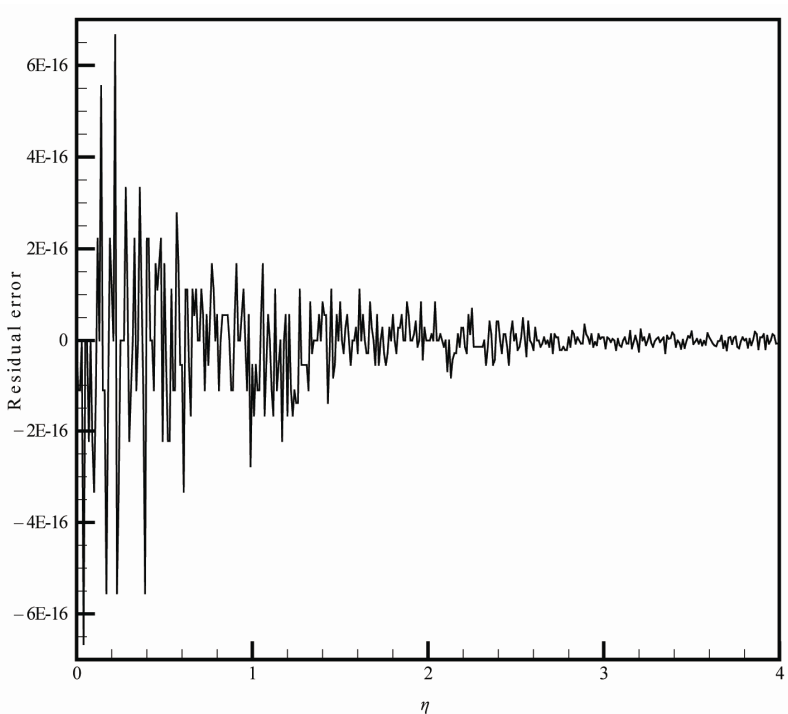

Figure 20. Residual error for Equation (15) using the MDTM approximations when $K=1, M=2, s=5$ and $a=-1$.

VIM, ADM and HPM). The method has been applied directly without requiring linearization, discretization, or perturbation. The accuracy of the method is excellent. The obtained results demonstrate the reliability of the algorithm and give it a wider applicability to non-linear differential equations.

\section{References}

[1] S. K. Ghosh, O. A. Bég, R. Bhargava, S. Rawat and T. A. Bég, "Mathematical Modelling of Transient Magnetohydrodynamic Couple Stress Biofluid Flow in a Rotating Channel," International Journal of Applied Mathematics and Mechanics, Vol. 6, No. 6, 2010, pp. 23-45.

[2] O. Anwar Bég, H. S. Takhar, R. Bharagava, S. Rawat and V. R. Prasad, "Numerical Study of Heat Transfer of a Third Grade Viscoelastic Fluid in Non-Darcian Porous Media with Thermophysical Effects," Physica Scripta, 2008, Vol. 77, No. , pp. 1-11.

[3] D. A. S. Rees and A. P. Bassom, "Boundary Layer Flow of a Micropolar Fluid,” International Journal of Engineering Science, Vol. 34, No. 1, 1996, pp. 113-124. doi:10.1016/0020-7225(95)00058-5

[4] O. A. Bég, A. Y. Bakier and V. R. Prasad, "Numerical Study of Free Convection Magnetohydrodynamic Heat and Mass Transfer from a Stretching Surface to a Saturated Porous Medium with Soret and Dufour Effects," Computational Materials Science, Vol. 46, No. 1, 2009, pp. 57-65. doi:10.1016/j.commatsci.2009.02.004

[5] S. K. Ghosh, O. Anwar Bég and M. Narahari, "Hall Effects on MHD Flow in a Rotating System with Heat Transfer Characteristics,” Meccanica, Vol. 44, No. 6, 2009, pp. 741-765. doi:10.1007/s11012-009-9210-6
[6] C. Truesdell and W. Noll, "The Non-Linear Field Theories of Mechanics,” In: S. Flügge, Ed., Encyclopedia of Physics, III/3, Springer, Berlin, 1965, pp. 1-591.

[7] K. R. Rajagopal, "On Boundary Conditions for Fluids of the Differential Type,” In: A. Sequeira, Ed., NavierStokes Equations and Related Non-linear Problems, Plenum Press, New York, 1995, pp. 273-278.

[8] K. R. Rajagopal and P. N. Kaloni, "Some Remarks on Boundary Conditions for Fluids of the Differential Type," In: G.A.C. Graham and S. K. Malik, Eds., Continuum Mechanics and Its Applications, Hemisphere, New York, 1989, pp. 935-942.

[9] K. R. Rajagopal and A. S. Gupta, "An Exact Solution for The Flow of a Non-Newtonian Fluid Past an Infinite Plate,” Meccanica, Vol. 19, No. 2, 1984, pp. 158-160. doi:10.1007/BF01560464

[10] Z. M. Odibat, C. Bertelle, M. A. Aziz-Alaoui, G. H. E. Duchamp, "A Multi-Step Differential Transform Method and Application to Non-Chaotic or Chaotic Systems," Computers and Mathematics with Applications, Vol. 59, No. 4, 2010, pp. 1462-1472. doi:10.1016/j.camwa.2009.11.005

[11] J. K. Zhou, "Differential Transformation and Its Applications for Electrical Circuits," Huazhong University of Science and Technology Press, Wuhan, 1986, (in Chinese).

[12] M. M. Rashidi and M. Keimanesh, "Using Differential Transform Method and the Padé Approximant for Solving MHD Flow in a Laminar Liquid Film from a Horizontal Stretching Surface,” Mathematical Problem in Engineering, Vol. 2010, Article ID 491319.

[13] M. M. Rashidi, M. Keimanesh, O. Anwar Bég and T. K. Hung, "Magnetohydrodynamic BioRheological Transport Phenomena in a Porous Medium: A Simulation of Magnetic Blood Flow Control and Filtration,” International Journal for Numerical Methods in Biomedical Engineering, Vol. 27, No. 6, 2011, pp. 805-821. doi:10.1002/cnm.1420

[14] M. M. Rashidi, "The Modified Differential Transform Method for Solving MHD Boundary-Layer Equations," Computer Physics Communications, Vol. 180, No. 11, 2009, pp. 2210-2217. doi:10.1016/j.cpc.2009.06.029

[15] I. H. Abdel-Halim Hassan, "Comparison differential Transformation Technique with Adomian Decomposition Method for Linear and Nonlinear Initial Value Problems," Chaos Solitons and Fractals, Vol. 36, No. 1, 2008, pp. 53-65. doi:10.1016/j.chaos.2006.06.040

[16] A. Robert Van Gorder, "High-order Nonlinear Boundary Value Problems Admitting Multiple Exact Solutions with Application to The Fluid Flow over a Sheet," Applied Mathematics and Computation, Vol. 216, No. 7, 2010, pp. 2177-2182. doi:10.1016/j.amc.2010.03.053

[17] M. M. Rashidi and H. Shahmohamadi, "Analytical Solution Of Three-Dimensional Navier-Stokes Equations for the Flow Near an Infinite Rotating Disk," Communications in Nonlinear Science and Numerical Simulation, Vol. 14, No. 7, 2009, pp. 2999-3006. doi:10.1016/j.cnsns.2008.10.030 
[18] M. M. Rashidi and S. Dinarvand, "Purely Analytic Approximate Solutions for Steady Three-Dimensional Problem of Condensation Film on Inclined Rotating Disk by Homotopy Analysis Method," Nonlinear Analysis: Real World Applications, Vol. 10, No. 4, 2009, pp. 2346-2356. doi:10.1016/j.nonrwa.2008.04.018
[19] M. M. Rashidi, S. A. Mohimanian pour and S. Abbasbandy, "Analytic Approximate Solutions for Heat Transfer of a Micropolar Fluid Through a Porous Medium with Radiation," Communications in Nonlinear Science and Numerical Simulation, Vol. 16, No. 4, 2011, pp. 1874-1889

$R$ suction parameter

$\lambda$ viscoelastic parameter

$v$ kinematic viscosity

$\sigma$ electric conductivity

$B$ uniform magnetic field along the $y$-axis

$M$ magnetic parameter 